\title{
Sozialtheoretische und begriffsgeschichtliche Aspekte des Problems der Beschleunigung der Geschichte. Überlegungen im Ausgang von Reinhart Koselleck
}

Falko Schmieder

Leibniz-Zentrum für Literatur- und Kulturforschung (Berlín)

schmieder@zfl-berlin.org - https://orcid.org/0000-0002-7977-8071

Fecha recepción: 29.05.2020 / Fecha aceptación: 26.06.2020

\section{Zusammenfassung}

Der Aufsatz versteht sich als Beitrag zur Erkundung der Aktualität und Grenzen von Reinhart Kosellecks begriffsgeschichtlichem Ansatz. Als Leitfaden soll dabei der Begriff der Beschleunigung dienen. Dieser Begriff hat sowohl für Kosellecks begriffsgeschichtliche wie auch für seine sozialtheoretischen Arbeiten eine besondere Bedeutung, denn er spielt eine zentrale Rolle in der Auseinandersetzung mit der Säkularisierungsde-

\begin{abstract}
The article aims to contribute to the exploration of the topicality and the limits of Reinhart Koselleck's approach to conceptual history. The concept of acceleration will serve as a guideline. It is of great relevance for Koselleck's conceptual history as well as for his sociological work, because it plays a central role in his intervention in the debate on secularization and is closely related to the concept of temporalization, which
\end{abstract}

* Der vorliegende Text ist im Rahmen des Forschungsprojekts FFI2017-82195-P der la AEI/FEDER, UE entstanden. 
Monográfico | Sozialtheoretische und begriffsgeschichtliche Aspekte des Problems der Beschleunigung der Geschichte.

batte und steht in enger Verbindung zum Begriff der Verzeitlichung, der das wohl wichtigste Interpretament in Kosellecks Deutung der sogenannten Sattelzeit war. Da es sich um eine sozial- und temporalanalytische Kategorie handelt, müsste sich die neuere, von Zeithistorikern und Soziologen vertretene These des Eintritts in eine postkoselleckianische Kultur nicht zuletzt anhand dieser Kategorie selbst entfalten lassen. Der Aufsatz diskutiert zunächst den Begriff der Beschleunigung und seine Implikationen bei Koselleck, um dann auf dieser Basis Bedeutungsdimensionen und Problemlagen zu entfalten, die über den Ansatz von Koselleck hinausweisen.

\section{Stichwörter}

Verzeitlichung, Beschleunigung, Säkularisierung, Begriffsgeschichte, Enthistorisierung, postkoselleckianische Kultur was probably the most important element in Koselleck's interpretation of the so-called saddle time. Since this is a social and time-analytical category, the more recent thesis of the entry into a post-Koselleckian culture, as advocated by contemporary historians and sociologists, should be able to unfold or be tested on the basis of this category itself. The paper first discusses the concept of acceleration and its implications for Koselleck, and then, on this basis, develops aspects and problems that go beyond Koselleck's approach.

\section{Keywords}

Temporalization, acceleration, secularization, conceptual history, dehistorization, post-Koselleckian culture 
Reinhart Kosellecks sozialgeschichtliche Begriffsgeschichte stößt heute auf weltweites Interesse. Sein Ansatz wird auf andere sprachliche und sozialhistorische Entwicklungen bezogen und dabei auch zwangsläufig modifiziert und erweitert. ${ }^{1}$ Aber auch in Deutschland ist speziell seit der Jahrtausendwende eine Historisierung Kosellecks, also eine Diskussion über die historischen Grenzen seines Ansatzes im Gange. War es eine der Ausgangshypothesen des Wörterbuchs der »Geschichtlichen Grundbegriffe«, »daß sich seit Mitte des 18. Jahrhunderts ein tiefgreifender Bedeutungswandel klassischer topoi vollzog, daß alte Worte neue Sinngehalte gewonnen haben, die mit Annäherung an unsere Gegenwart keiner Übersetzung mehr bedürftig sind «, ${ }^{2}$ so wurde in jüngerer Zeit die These vertreten, »dass viele jener, in der Sattelzeit "neu gewonnenen Sinngehalte" heute nicht mehr ohne Übersetzung auskommen, und dass ihre damalige Herkunft nicht mehr unmittelbar in "unsere Präsenz" mündet. «" Positiv gewendet heißt das, dass die Transformationen, die in die Moderne führten, sich in einer Transformation der Moderne fortsetzten. ${ }^{4}$ Das Wörterbuch der »Geschichtlichen Grundbegriffe« verfolgt die meisten Begriffe nur bis ins erste Drittel des 20. Jahrhunderts. Die neuere Begriffs- und Zeitgeschichte geht nun davon aus, dass wir seit den 1970er Jahren in eine, wie der Historiker Paul Nolte formuliert, "postkoselleckianische« Kultur eingetreten sind, wobei es sich allerdings um einen Umbruch handelt, dessen Deutung vorerst nur skizzenhaft möglich ist. ${ }^{5}$ Ein sprachlicher Indikator dieses Umbruchs ist die Vorsilbe »post«, die

1. Vgl. zur internationalen Rezeption des Werkes von Koselleck E. Müller (Hg.), Forum Interdisziplinäre Begriffsgeschichte (E-Journal), 4. Jahrgang, Heft 1 (2015); https://www.zfl-berlin.org/files/zfl/downloads/ publikationen/forum_begriffsgeschichte/ZfL_FIB_4_2015_1.pdf; vgl. auch E. Müller und F. Schmieder, Begriffsgeschichte und historische Semantik. Ein kritisches Kompendium, Suhrkamp, Berlin 2016, S. 801-819.

2. R. Koselleck, »Einleitung", in: Geschichtliche Grundbegriffe. Historisches Lexikon zur politisch-sozialen Sprache in Deutschland, hg. v. R. Koselleck, O. Brunner und W. Conze, 8 Bde., Klett-Cotta, Stuttgart 19721997, Bd. 1, S. XIVf.

3. Chr. Geulen: »Plädoyer für eine Geschichte der Grundbegriffe des 20. Jahrhunderts«, in Zeithistorische Forschungen, H. 1 (2010), S. 79-97, hier S. 81.

4. Vgl. ebd. - Zur allgemeineren Debatte vgl. K. Kollmeier und St.-L. Hoffmann: »Geschichtliche Grundbegriffe Reloaded? Writing the Conceptual History of the Twentieth Century. Roundtable Discussion «, in Contributions to the History of Concepts 7 (2012) 2, S. 78-128.

5. P. Nolte: »Vom Fortschreiben und Umschreiben der Begriffe. Kommentar zu Christian Geulen«, in Zeithistorische Forschungen 7 (2010) 1, S. 98-103, hier S. 101. Zum semantischen Umbruch der 1970er Jahre 
vor allem seit den 1970er Jahren vielen klassischen Begriffen vorgeschaltet wird - man denke etwa an postindustriell, Postmoderne, Posthistoire, Posthumanismus oder Postdemokratie.

Der vorliegende Aufsatz versteht sich als Beitrag zur Erkundung dieses Umbruchs, und damit zugleich auch der Aktualität und Grenzen von Kosellecks begriffsgeschichtlichem Ansatz. Als Leitfaden soll dabei der Begriff der Beschleunigung dienen, der für Koselleck sowohl begriffsgeschichtlich wie auch sozialtheoretisch eine besondere Bedeutung hat. Er spielt eine zentrale Rolle in Kosellecks Beitrag zur Säkularisierungsdebatte ${ }^{6}$, darüber hinaus steht er aber auch in enger Verbindung zum Begriff der Verzeitlichung, der das wohl wichtigste Interpretament in Kosellecks Deutung der sogenannten Sattelzeit war. In dieser Verbindung ist der Begriff der Beschleunigung zugleich Teil einer Theorie historischer Zeiten und übergreifend einer Theorie der modernen Gesellschaft. In diesem Rahmen spricht Koselleck sogar davon, dass ihm der Begriff der Beschleunigung als ein "Erkenntnisinstrument dient, das auf eine politische Krisenlehre zielt. ${ }^{7} \mathrm{Da}$ es sich um eine sozial- und temporalanalytische Kategorie handelt, müsste sich die These des Eintritts in eine postkoselleckianische Kultur nicht zuletzt anhand dieser Kategorie selbst entfalten oder prüfen lassen, was im Folgenden näher gezeigt werden soll.

Die Argumentation gliedert sich in drei Teile: Im ersten Teil sollen der Begriff der Beschleunigung und seine Implikationen bei Koselleck diskutiert werden, um auf dieser Basis dann im zweiten Teil im Ausgang von diesem Begriff Bedeutungsdimensionen und Problemlagen zu entfalten, die über den Ansatz von Koselleck hinausweisen. Im dritten Teil schließlich werden die Beobachtungen resümiert und die problemgeschichtliche Kontinuität zu Kosellecks Fragestellungen einer sozialgeschichtlich informierten Begriffsgeschichte herausgestellt.

vgl. A. Doering-Manteuffel und L. Raphael: Nach dem Boom. Perspektiven auf die Zeitgeschichte seit 1970, Vandenhoeck\&Ruprecht, Göttingen 2008. Der Forschungsverbund »Nach dem Boom« hat seit 2008 mehrere Fallstudien erarbeitet, vgl. u. a. M. Reitmayer und Th. Schlemmer (Hg.): Die Anfänge der Gegenwart. Umbrüche in Westeuropa nach dem Boom, Oldenbourg, München 2014; A. Doering-Manteuffel, L. Raphael und Th. Schlemmer (Hg.): Vorgeschichte der Gegenwart. Dimensionen des Strukturbruchs nach dem Boom, Vandenhoeck\&Ruprecht, Göttingen 2016. Vgl. weiter die Beiträge in A. Leendertz und W. Meteling (Hg.): Die neue Wirklichkeit. Semantische Neuvermessungen und Politik seit den 1970er-Jahren, Campus, Frankfurt a. M./New York 2016; U. Bröckling, S. Krasmann und Th. Lemke (Hg.), Glossar der Gegenwart, Suhrkamp, Frankfurt/M. 2004; St. Lessenich (Hg.), Wohlfahrtsstaatliche Grundbegriffe. Historische und aktuelle Diskurse, Campus, Frankfurt/M., New York 2003.

6. R. Koselleck, »Zeitverkürzung und Beschleunigung. Eine Studie zur Säkularisation «, in: ders., Zeitschichten. Studien zur Historik, Suhrkamp, Frankfurt/M. 2000, S. 177-202.

7. R. Koselleck, »Gibt es eine Beschleunigung der Geschichte?«, in: ders., Zeitschichten, S. 150-176, hier S. 165. 
Der Begriff der Beschleunigung spielt für das Werk von Koselleck eine wichtige Rolle. ${ }^{8}$ Anders als der Begriff der Verzeitlichung, den Koselleck für die semantischen Transformationsprozesse seit der Neuzeit um 1500 verwendet $^{9}$, reserviert Koselleck den Begriff der Beschleunigung ausschließlich für die Entwicklungen der Moderne, die mit der politischen und industriellen Doppelrevolution um 1800 beginnt. ${ }^{10}$ Ein quellensprachlicher Ausgangspunkt für diese Differenzierung ist die historiographische Unterscheidung von Neuzeit und neuester Zeit. Wie Koselleck ausführt, wird seit dem 18. Jahrhundert in der Geschichtsschreibung der Ausdruck der neuen Zeit bzw. der Neuzeit verwendet. ${ }^{11}$ Herrscht zunächst eine unspezifische Bedeutung vor, so schält sich dann in der Aufklärung allmählich eine epochenspezifische Bedeutung heraus. Das Ereignis der Französischen Revolution, das von den Zeitgenossen unmittelbar als eine historische Zäsur erfahren wurde, setzte dann den Begriff der neuesten Zeit frei. Mit diesem Begriff gelang nun, was mit dem Begriff der neuen Zeit noch nicht möglich war: die neueste Zeit wurde zu einem zeitgenössischen Epochenbegriff, der nicht nur rückblickend eine neue Periode registrierte, sondern der eine in die Zukunft weisende neue Periode eröffnete. ${ }^{12}$ Die Differenzbestimmung zwischen der neuen und der neuesten Zeit war verbunden mit einer Intensivierung der Reflexionen über die geschichtliche Zeit. Im Zuge dieser Reflexionen gewannen die modernen Kollektivsingulare wie der Fortschritt, die Revolution oder die Geschichte ihr Profil. Bei allen diesen Begriffen handelt es sich um selbstbezügliche, zukunftsoffene Bewegungsbegriffe. Mit ihrer Verwendung artikulierten die Zeitgenossen der neuesten Zeit die Erfahrung, in einer permanenten Übergangszeit zu leben, in der immer wieder Neues und Unerwartetes zu Tage tritt, das nicht aus den überlieferten Erfahrungen ableitbar ist. Der alte Lehrsatz »Historia Magistra Vitae« verliert unter diesen Bedingungen seine Plausibilität und Bedeutung. ${ }^{13}$ Das neue

8. Vgl. H. Rosa, Beschleunigung. Die Veränderung der Zeitstrukturen in der Moderne, Suhrkamp, Frankfurt/M. 2005, der sich sowohl in Bezug auf seine Quellen wie auch seine zeittheoretischen Überlegungen stark auf Koselleck stützt; vgl. auch P. Osborne, The Politics of Time. Modernity and Avant-Garde, Verson London, New York 1995, bes. S. 9-14; 122-123.

9. Vgl. den historischen Einsatz mit Francis Bacon in: R. Koselleck, »Zeitverkürzung und Beschleunigung. Eine Studie zur Säkularisation, in ders., Zeitschichten, a.a.O., S. 177-202, hier S. 188. Seinem Aufsatz "Historia Magistra Vitae. Über die Auflösung des Topos im Horizont neuzeitlich bewegter Geschichte", in ders., Vergangene Zukunft. Zur Semantik geschichtlicher Zeiten, Suhrkamp, Frankfurt/M. 1989, S. 38-66 stellt Koselleck ein Zitat von Shakespeare als Motto voran, vgl. ebd., S. 38.

10. Vgl. R. Koselleck, ", Neuzeit'. Zur Semantik moderner Bewegungsbegriffe«, in: ders., Vergangene Zukunft, S. 300-348; vgl. ders., »Wie neu ist die Neuzeit? «, in ders., Zeitschichten, S. 225-239.

11. Vgl. dazu Koselleck, ", Neuzeit" «.

12. Vgl. ebd., S. 320.

13. Vgl. Koselleck, »Historia Magistra Vitae«. 
Epochenbewusstsein reflektiert sich auch in dem neuen Verständnis von historischer Zeit: "Zeit bleibt nicht länger die äußere Form, in der sich alle Geschichten abspielen, sondern sie gewinnt selber eine geschichtliche Qualität: Nicht mehr in der Zeit, sondern durch die Zeit vollzieht sich dann die Geschichte. Die Zeit wird dynamisiert zu einer Kraft der Geschichte selber « ${ }^{14}$, die nun als selbstbezüglicher Kollektivsingular angesprochen wird. Geschichte stellt sich so dar als Sukzession von Präzendenzlosigkeiten. Koselleck hat diese an die Epoche der neuesten Zeit geknüpften grundstürzenden Veränderungen des Zeitbewusstseins unter anderem anhand des Revolutionsbegriffs näher analysiert. ${ }^{15}$ Ursprünglich ein naturaler, zyklischer, am Umlauf der Sterne orientierter Begriff, nimmt er in der Moderne die entgegengesetzte Bedeutung an: er bezieht sich jetzt auf einen gesellschaftlichen Prozess, der in eine offene, unbekannte Zukunft führt. Signalgebend war zunächst besonders die Französische Revolution; im ersten Drittel des 19. Jahrhunderts entstehen dann aber bereits politökonomische Begriffe wie »industrielle Revolution $\aleph^{16}$ oder $»$ revolutionäre Entwicklung «. Bemerkenswert an diesen Prägungen ist die mit ihnen bewerkstelligte Übertragung von Gehalten einer punktuellen, als gewaltsam, umstürzend oder katastrophal angesehenen politischen Ausnahmesituation in den Kontext der alltäglichen Reproduktion der gesellschaftlichen Ordnung. Um die Mitte des 19. Jahrhunderts werden diese Begriffe dann schon selbst zu Kernbestimmungen einer spezifischen Epochenerfahrung: Karl Marx und Friedrich Engels beschreiben im »Manifest der Kommunistischen Partei« die Bourgeoisieepoche als permanente Revolutionierung und Umwälzung der gesellschaftlichen Zustände $^{17}$; Friedrich Julius Stahl sieht in einem Vortrag aus dem Jahre 1852 in der Revolution die eigentümliche weltgeschichtliche Signatur unseres Zeitalters ${ }^{18}$; Jacob Burckhardt spricht von einem revolutionären Weltalter ${ }^{19}$ - die Beispiele ließen sich um viele vermehren. Metaphorologisch drückt sich die im Begriff der neuesten Zeit erfasste moderne Epoche in neuen Sprachbildern aus, die Gesellschaft als Schicksalszusammenhang einer zweiten Na-

14. Koselleck, »,Neuzeit" «, S. 321

15. R. Koselleck, "Historische Kriterien des neuzeitlichen Revolutionsbegriffs«, in ders., Vergangene Zukunft, S. 67-86.

16. Die Angaben zur Entstehung dieses Begriffs in den Geschichtlichen Grundbegriffen sind widersprüchlich: Lucian Hölscher gibt in seinem Beitrag zum Artikel »Industrie« als frühesten Beleg für »industrielle Revolution« einen aus dem Jahre 1827 an, vgl. Hölscher, [Art.] »Industrie, Gewerbe«, in: Geschichtliche Grundbegriffe, Bd. 5, Stuttgart 2004, S. 237-304, hier S. 294; in dem von Koselleck verfassten Artikel »Revolution« dagegen wird der erste Beleg auf das Jahr 1797 datiert, vgl. Koselleck, [Art.] »Revolution. Rebellion, Aufruhr, Bürgerkrieg«, in: Geschichtliche Grundbegriffe, Bd. 5, S. 653-788, hier S. 769. Vgl. dazu A. Bezanson: »The Early Use of the Term Industrial Revolution«, in The Quarterly Journal of Economics, Vol. 36, No. 2 (1922), S. 343-349.

17. Vgl. K. Marx und Fr. Engels: »Manifest der Kommunistischen Partei«, in dies.: Werke, hg. vom Institut für Marxismus-Leninismus, Bd. 4, Dietz, Berlin (Ost) 1971, S. 459-493, hier S. 465.

18. F. J. Stahl, »Was ist die Revolution? Ein Vortrag auf Veranstaltung des Evangelischen Vereins für kirchliche Zwecke, am 8. März 1852 gehalten«, W. Schultze, Berlin 1852, S. 3.

19. Jacob Burkhardt, zitiert nach F. Stern, Das feine Schweigen. Historische Essays, Beck, München 1999, S. 17. 
tur erfassen: der Prozess der Geschichte erscheint als Strudel, Strom, Sturm oder Erdbeben. Auch die Allegorien für Geschichte halten die Selbstbezüglichkeit des Prozesses und den damit verbundenen Kontrollverlust sowie die Entfremdungserfahrung fest: prägnant erscheint in diesem Zusammenhang etwa Goethes Prägung des Veloziferischen, in der das Bewegungsmotiv mit dem Teuflischen liiert ist ${ }^{20}$; andere Allegorien für moderne Geschichte sind Goethes Figur des Zauberlehrlings ${ }^{21}$ oder Hegels Furie des Verschwindens. ${ }^{22}$

Koselleck stellt nun aber nicht nur die Verzeitlichung der Geschichte heraus, sondern diese Verzeitlichung selber erhält bei ihm einen zeitlichen Index, wenn sie unter der Bestimmung der Beschleunigung gefasst wird. Es geht also nicht nur um Veränderungen, sondern auch um die Temporalisierung der Veränderung, ihre Steigerung bzw. Beschleunigung. ${ }^{23}$ Der Begriff der Beschleunigung gehört zum Sprachgebrauch der neuesten Zeit, die mit seiner Hilfe auch den Unterschied zur neuen Zeit erfasst hat. Zur neuen Zeiterfahrung der Moderne gehört es nämlich auch, dass sich alles schneller ändern kann, als man bisher erwarten konnte oder früher erfahren hatte. Bereits Joseph Görres registrierte im ersten Drittel des 19. Jahrhunderts die beständige Verkürzung der Perioden, wie sie der Gegenwart näher kommen, und Werner von Siemens postuliert dann im Jahre 1886 das sogenannte Beschleunigungsgesetz: Entwicklungsperioden, die in früheren Zeiten erst in Jahrhunderten durchlaufen wurden, die im Beginne unserer Zeitperiode noch der Jahrzehnte bedurften, vollenden sich heute in Jahren und treten häufig schon in voller Ausbildung ins Dasein. $\star^{24}$ Durch die Beschleunigung, so Koselleck, verkürzen sich beständig die Zeitspannen, innerhalb derer mit neuen, präzedenzlosen Erfahrungen zu rechnen ist. Mit der Temposteigerung wird es zudem immer schwieriger, zukünftige Ereignisse vorherzusagen oder zu antizipieren. An dieser Stelle schlagen Kosellecks begriffsgeschichtliche Überlegungen dann in geschichts- und sozialtheoretische um. Die geschichtliche Steigerung des Veränderungskoeffizienten bedeutet eine beständige Transformation der sozialen Verhältnisse, die unausweichlich mit Prozessen der Instabilisierung und Desintegration verbunden ist und zu regelmäßig wiederkehrenden krisenhaften Zuspitzungen führt. ${ }^{25}$ Analytisch kann der Begriff der Beschleunigung daher als ein Erkenntnisinstrument $«$ verwendet werden, "das auf eine politische Krisenlehre zielt. ${ }^{*}$

20. M. Osten, ,Alles veloziferisch oder Goethes Entdeckung der Langsamkeit. Zur Modernität eines Klassikers im 21. Jahrhundert, Insel, Frankfurt/M. 2003.

21. Diese Figur ist theoriegeschichtlich sehr wirkmächtig geworden und bildet einen integralen Bestandteil vieler Technik- oder Moderntheorien, vgl. u.a. N. Wiener: „Some Moral and Technical Consequences of Automation «, in Science 131 (1960), 1355-1358; G. Anders, Die Antiquiertheit des Menschen 2. Uber die Zerstörung des Lebens im Zeitalter der dritten industriellen Revolution, Beck, München 1980, 396-405; U. Beck: Die Welt als Labor «, in: ders. (Hg.), Politik in der Risikogesellschaft, Suhrkamp, Frankfurt/M. 1987, S. 154-166.

22. Vgl. G. W. F. Hegel, Phänomenologie des Geistes, Suhrkamp, Frankfurt/M. 1977, S. $435 f$.

23. Vgl. Koselleck,, Zeitverkürzung und Beschleunigung $\alpha$.

24. Vgl. Koselleck, »Zeitverkürzung und Beschleunigung «, S. 178.

25. Joseph Schumpeter hat mit seinem Verständnis des Kapitalismus als Prozess der schöpferischen Zerstörung ein wirkmächtiges Theorem geliefert, das zugleich als Variation oder Konkretisierung des Theorems 
Im Bereich der geschichtszeitlichen Beschleunigung zeichne sich die Möglichkeit ab, »dass der Mensch selber die überkommenen, kulturell und industriell angereicherten Bedingungen seiner Existenz vernichtet. ${ }^{26}$ Speziell in der ökologischen Krise sah Koselleck ein Konfliktpotential, an dem er das auf Carl Schmitt zurückgehende Verständnis von Krise als einer »Letztentscheidung « ${ }^{27}$ erneuerte. »Offenbar sind Entscheidungen fällig, die, wissenschaftlich oder nicht, gewollt oder ungewollt, darüber befinden, ob und wie das Überleben auf diesem Globus möglich ist oder nicht. $\ll^{28}$ Mit der an der Club of Rome-Studie »Grenzen des Wachstums « orientierten These, »dass bestimmte Beschleunigungsvorgänge in unserer ausdifferenzierten Gesellschaft ihren Sättigungsgrad erreicht haben ${ }^{29}$, visiert Koselleck auch ein mögliches Ende der modernen Geschichtsdynamik und damit die Konturen einer anderen Gesellschaftsform, die auch der Bericht des Club of Rome angedeutet hatte. Konsequenterweise stellt Koselleck dann auch die Frage nach den Triebkräften des permanenten Wandels, die er in den technischen und industriellen Entwicklungen sieht. Diese Einschätzung ist sicher unzulänglich und verweist auf das Desiderat einer Theorie der kapitalistischen Gesellschaft. Sie reicht aber hin, um das in den Geisteswissenschaften verbreitete Säkularisierungstheorem zu kritisieren, wie es prononciert Karl Löwith vertreten hatte. Während dieser nämlich im Fortschritt eine Säkularisierung eschatologischer Vorstellungen sah, hielt Koselleck entgegen, »dass der harte Kern der modernen Beschleunigungserfahrung, nämlich die technische und industrielle Überformung der menschlichen Gesellschaft, selber nicht mehr aus theologischen Prämissen ableitbar ist. « ${ }^{30}$

Nach diesen Ausführungen soll nun im zweiten Teil der Frage nach den historischen Grenzen von Kosellecks Ansatz und der These des Eintritts in eine sogenannte postkoselleckianische Kultur nachgegangen werden. Wie aus dem Entwickelten hervorgeht, ist die moderne Gesellschaft durch eine permanente Selbstüberholung charakterisiert. Ihre Identität erhält sich also gerade im auf Dauer gestellten Wandel; sie ist stabil, wenn sie expandiert. Auf einer allgemeinen sprachlichen Ebene drückt sich diese Paradoxie in einer Reihe von Wendungen aus, die

der industriellen Revolution bzw. der permanenten Revolution angesehen werden kann; vgl. ders. Capitalism, Socialism and Democracy, Harper, New York 2008 [1942], bes. S. 81-87.

26. Koselleck, "Zeitverkürzung und Beschleunigung", S. 200.

27. R. Koselleck, "Einige Fragen an die Begriffsgeschichte von "Krise“", in: ders., Begriffsgeschichten, S. 203-217, hier S. 212, 213, 216.

28. Ebd., S. 215.

29. Koselleck, »Zeitverkürzung und Beschleunigung«, S. 199.

30. Ebd., S. 195. Diese Formulierung Kosellecks läßt es fraglich erscheinen, ob er sich in der Kontroverse zwischen Löwith und Blumenberg »eindeutig auf Löwiths Seite" gestellt hat, vgl. H. Joas: „Die Kontingenz der Säkularisierung. Überlegungen zum Problem der Säkularisierung im Werk Reinhart Kosellecks«, in: H. Joas und P. Vogt (Hg.), Begriffene Geschichte. Beiträge zum Werk Reinhart Kosellecks, Suhrkamp, Frankfurt/M. 2011, S. 319-338, hier S. 328. 
zwar Veränderung und Steigerung behaupten, sich aber in ihrer sprachlichen Form als unüberbietbar erweisen, wie etwa »immer schneller«, »immer mehr« oder »zunehmend «. Die neuen geschichtlichen Dimensionen, auf die diese Wendungen hindeuten, lassen sich nur über die damit jeweils bezeichneten konkreten Gehalte und Kontexte erfassen. Ein hervorstechender Zug speziell seit der zweiten Hälfte des 20. Jahrhunderts ist die Häufung von Epochen- bzw. Gesellschaftsbestimmungen, die jeweils einen als neu und prägend empfundenen technischen oder kulturellen Zug totalisieren und tiefgreifende kollektive Erfahrungsumbrüche indizieren - dazu gehören Begriffe wie "Atomic Age«, Computerzeitalter, Informationszeitalter, Postindustrielle Gesellschaft, Dienstleistungsgesellschaft, „Cyber Age« oder die normativen, stärker zukunftsbezogenen Begriffe Solarzeitalter oder »Low-Carbon-Society«. Diese Häufung neuer Epochenbezeichnungen lässt sich als Beschleunigung der Zeitwendeerfahrungen verstehen, die Koselleck in seiner Reflexion der Differenzen von neuer und neuester Zeit analysiert hatte. ${ }^{31}$ Mit den Epochenentwürfen gehen zugleich neue Formen der Selbstbeschreibung einher, die ihre eigenen Vokabulare prägen. Eine parallele Erscheinung der Beschleunigung der Zeitwendeerfahrungen speziell seit der zweiten Hälfte des 20. Jahrhunderts ist die Charakterisierung markanter zeitgenössischer Ereignisse als historisch (»historischer Moment«, »historisches Ereignis«). Sie deutet auf eine Aufspreizung des jeweiligen Gegenwartsbewusstseins hin, die in Geschichtsverlust umzuschlagen droht. Oft verweisen solche als historisch charakterisierten Ereignisse auf politische Regimewechsel oder Durchbrüche technischer oder naturwissenschaftlicher Entwicklungen, immer öfter aber auch auf niedrigschwellige zeitgeschichtliche Überraschungsmomente. In sozialgeschichtlicher Perspektive lassen sich solche Instantanhistorisierungen in der Kontinuität des um 1800 emergierenden Bewusstseins verstehen, in einer permanenten Übergangszeit zu leben. Hatte Koselleck als Motoren dieses neuen Zeitbewusstseins die politisch-industrielle Doppelrevolution (und gelegentlich die Entwicklung der Technik) gesehen, so übernimmt im 20. Jahrhundert die zur Produktivkraft gewordene Wissenschaft eine Treiberfunktion für gesellschaftlichen und damit auch sprachlichen Wandel. ${ }^{32}$ In der neueren Begriffsgeschichte hat sich deshalb auch die heuristische Leithypothese einer Verwissenschaftlichung des Sprachgebrauchs bewährt. Komplementär zur These von der »Verwissenschaftlichung des Sozialen $\aleph^{33}$ ist in der Perspektive des weiteren Jahrhundertverlaufs zugleich auch eine aus ihrem vertieften Anwendungsbezug und praktischen Folgen resultierende Politisierung und Sozialisierung der Natur- und Technikwissenschaften in Rechnung zu stellen.

31. Zum Begriff der Zeitwende vgl. auch Richard Koebner, der für Koselleck sicher von Bedeutung war: R. Koebner, »Die Idee der Zeitwende«, in ders., Geschichte, Geschichtsbewußtsein und Zeitwende. Vorträge und Schriften aus dem Nachlaß, hg. v. Institut für Deutsche Geschichte der Universität Tel Aviv, Gerlingen 1990, S. 147-193; vgl. auch P. Tietze: "Zeitwende“: Richard Koebner und die Historische Semantik der Moderne«, in: Jahrbuch des Simon-Dubnow-Instituts 13 (2014), S. 131-165.

32. Vgl. D. Kaldewey, und D. Schauz (Hg.), Basic and Applied Research. The Language os Science Policy in the Twentieth Century, Berghahn, New York, Oxford 2018.

33. L. Raphael: »Die Verwissenschaftlichung des Sozialen als methodische und konzeptionelle Herausforderung für eine Sozialgeschichte des 20. Jahrhunderts«, in: Geschichte und Gesellschaft 22 (1996), H. 2, S. $165-193$. 
$\mathrm{Zu}$ den neuen Herausforderungen gehört daher eine engere Verbindung der Begriffsgeschichte mit der Wissenschaftsgeschichte, die bei Koselleck und in den "Geschichtlichen Grundbegriffen « noch weithin ausgespart ist. Eine Pointe der Verwissenschaftlichungsthese besteht darin, dass damit die Begriffsgeschichte methodisch eine (im Vergleich mit dem für Koselleck zentralen 18. und 19. Jahrhundert) noch größere Plausibilität erhält, weil begriffsförmiges, rationalisiertes und spezialisiertes Wissen für die Reproduktion der Gesellschaft insgesamt an Bedeutung gewinnt. Die gewachsene soziale Bedeutung der Wissenschaften und der mit ihr verbundene erhöhte Aufwand an Technik führen zu einer »Futurisierung « der Forschung, deren Folgen immer weiter in die Zukunft übergreifen. Die sich allmählich auch international verfestigende Verbindung von Kosellecks begriffsgeschichtlichem Ansatz mit dem der Wissenschaftsgeschichte ${ }^{34}$ kann so auch als überfälliger Effekt einer veränderten Beziehung von Gesellschaft und Wissenschaft betrachtet werden, wie sie in Begriffen wie Risikogesellschaft, Wissensgesellschaft, High-Tech-Capitalism, Anthropozän, Technozän oder im Verständnis der Globalgeschichte unter dem Begriff des Realexperiments ${ }^{35}$ impliziert ist.

Aus der Verwissenschaftlichung und dem verschärften internationalen Forschungswettbewerb ergibt sich ein weiteres Paradox, denn es ist gerade die Erfahrung der Dynamisierung und Beschleunigung der Geschichte, die zu einem Abschmelzen der historischen Dimensionen der Begriffe und zu einer beschleunigten semantischen Veralterung führt. ${ }^{36}$ In Bezug auf die im Wörterbuch der »Geschichtlichen Grundbegriffe» betonte longue durée der semantischen Vorgaben der modernen Begriffe fällt auf, dass viele der gegenwärtigen Grundbegriffe jüngeren Datums und oft erst deutlich nach der Sattelzeitperiode entstanden sind, wie etwa der Begriff Ökologie, der erstmals in Ernst Haeckels »Generelle Morphologie der Organismen« aus dem Jahre 1866 auftaucht und dann für längere Zeit in dem eingeführten Sinne als Fachterminus einer biologischen Teildisziplin verwendet wurde, ${ }^{37}$ bevor er dann über ein Jahrhundert später im Zusammenhang der Umweltproblematik disziplinär entgrenzt zu einem politisch aufgeladenen globalen umbrella term aufrückte. Ähnlich verläuft die Karriere des Begriffs Nachhaltigkeit. Die geringe historische Tiefenschicht vieler neuer Schlüsselbegriffe zeigt sich darin, dass sie in den umfangreichen Registerbänden der »Geschichtlichen Grundbegriffe" entweder gar nicht (wie Diversität, Globalisierung, Innovation, Regulierung, Ressource) oder nur sehr selten (wie Information, Interaktion, Kommunikation, Medien, Umwelt) auftauchen. Vielleicht lässt sich hieran die Tendenz einer abnehmenden Beständigkeit bzw. schnelleren Verfallszeit von Begriffen ablesen, wie sie einer sich beschleunigenden Geschichtsdynamik entspricht.

34. Vgl. Robert Bud: »Framed in the Public Sphere: Tools for the Conceptual History of ,Applied Science - A Review Paper", in History of Science 51 (2013) 4, S. 413-433.

35. Vgl. F. Schmieder: "Geschichte als Realexperiment. Problem und Metaphorik der Unverfügbarkeit«, in Zeitschrift für Kulturphilosophie, 8. Jg. (2014), Heft 1, S. 35-46.

36. Vgl. H. Lübbe, Im Zug der Zeit. Verkürzter Aufenthalt in der Gegenwart, Springer, Berlin u.a. 1992.

37. E. Haeckel, Generelle Morphologie der Organismen. Allgemeine Grundzüge der organischen Formen-Wissenschaft, mechanisch begründet durch die von Charles Darwin reformirte Descendenz-Theorie, Reimer, Berlin, 1866; Bd. 2, S. 286. 
Durch diese Beschleunigung des semantischen Wandels nähert sich die Begriffsgeschichte in verstärktem Maße der Zeitgeschichte und der Politikwissenschaft sowie der Wissenschaftsgeschichte an. ${ }^{38}$ Mit der wachsenden gesellschaftlichen Bedeutung der Natur- und Technowissenschaften wandelt sich auch der Begriff des Politischen selbst. Der strukturell wohl deutlichste Wandel in der Semantik ist die Herausbildung einer Vielzahl von Entwicklungs- und Prozesskategorien mit der Endung -isierung (engl.: -ication), die im 20. Jahrhundert den für Koselleck noch zentralen Bewegungsbegriffen mit der Endung -ismus den Rang ablaufen - zu nennen sind etwa die Begriffe Globalisierung, Digitalisierung, Technisierung, Medialisierung, Ökonomisierung oder der Begriff der Modernisierung, der den Begriff der Moderne prozessualisiert bzw. verflüssigt. Solche Leitbegriffe, wie sie Koselleck selbst auf der theoretisch-reflexiven Metaebene zur Analyse der übergreifenden semantischen Prozesslogiken verwendet hatte (Verzeitlichung, Demokratisierung, Politisierung), tauchen wohl auf breiterer Front überhaupt erst im 20. Jahrhundert auf. Dies kann als weiterer Beleg für die Plausibilität der Verwissenschaftlichungsthese verstanden werden und dokumentiert den gewachsenen Abstraktionsgrad der Begriffe und das gesteigerte gesellschaftliche Bedürfnis nach wissenschaftlicher Selbstdeutung. Offenbar handelt es sich bei diesen Prozessbegriffen um eine neue Kategorie systemisch-selbstbezüglicher Kollektivsingulare. Während die traditionellen Kollektivsingulare wie Fortschritt, Revolution oder Geschichte noch stark an die Geschichtsphilosophie gebunden sind, reflektiert sich in den neuen Kollektivsingularen die Ausdifferenzierung gesellschaftlicher Teilsysteme, die zwar ineinander verflochten sind, aber zugleich auch eigendynamische Evolutionen durchmachen. In der Begriffsgeschichte ist der spezifische Status dieser Kategorien bislang noch kaum erforscht. Vielleicht deutet sich heute an den Grenzen des Wohlfahrtsstaates das Auseinanderbrechen der in der verwissenschaftlichten Politik zum Ausdruck kommenden Rationalitätsform an: Diagnosen einer Depotenzierung traditioneller Formen von Politik, auf die neuere Begriffe wie Postdemokratie, Postfaktische Politik, "Fake News«, "Alternative Facts« oder Populismus zielen, stehen solchen ihrer manageriell-technokratischen Verdinglichung gegenüber, wie sie in Stichwörtern wie Autoritarismus, gelenkte Demokratie, Technokratie, Expertokratie, Alternativlosigkeit oder Sachzwang angedeutet ist. Nach dem tiefgreifenden Strukturwandel der Öffentlichkeit, wie ihn etwa Jürgen Habermas beschrieben hat, ${ }^{39}$ ist in Bezug auf die Semantik für das 20. Jahrhundert sicher eine gewachsene Bedeutung des Einflusses von institutionalisierten Interessenvertretungen wie Public-Relation- und Lobbyverbänden sowie professioneller Think-Tanks in Rechnung zu stellen. Aktuell frappiert die Vielzahl von Euphemismen im Sprachgebrauch der Politik, wie sie etwa in der im Zusammenhang der Verschärfung der Flüchtlingskrise zu beobach-

38. Vgl. K. Palonen: "Begriffsgeschichte und/als Politikwissenschaft», in Archiv für Begriffsgeschichte 44 (2002) S. 221-234.

39. Vgl. J. Habermas, Strukturwandel der Öffentlichkeit. Untersuchungen zu einer Kategorie der bürgerlichen Gesellschaft [1962], Luchterhand, Darmstadt, Neuwied 1982. 
tenden Ersetzung des Ausdrucks Lager durch eine Fülle anderer Begriffe wie Begrüßungszentrum, Transitzone oder Hotspot zu beobachten ist.

Methodisch ergibt sich mit Blick auf die neuen Medien und ihre internationale bzw. globale Reichweite, dass heute neue Formate und Prozesse der beschleunigten Ausbreitung von Begriffen in Rechnung zu stellen sind. Ein wichtiges Charakteristikum der neuen geschichtlichen Grundbegriffe ist »die Form ihres Aufstiegs zu Leitbegriffen«, der nur in den seltensten Fällen präzise rekonstruierbar ist. »Eine allmähliche Wanderung dieser Begriffe von einem Feld ins andere oder von ihrem Ursprung in verschiedene Anwendungsgebiete ist kaum nachzuzeichnen. Ihre Ausbreitung ist weniger als Rezeption denn als multiple Projektion beschreibbar. « ${ }^{40}$ Zeittheoretisch neu zu bedenken wären auch die Kontinuitäten, Brüche und Schwellen von Begriffsentwicklungen. Kosellecks Konzept der Sattelzeit war auf einen ca. hundert Jahre umspannenden Zeitraum bezogen, in Bezug auf den er einander wechselseitig stützende Trends formulierte, deren markantester die Verzeitlichung der Sprache ist. Ungeachtet seiner großen Plausibilität und empirischen Evidenz ist der Ansatz der "Geschichtlichen Grundbegriffe« dennoch hochgradig selektiv und mit etlichen Problemen verbunden. In Bezug auf das 19. Jahrhundert ist etwa festzuhalten, dass die beiden komplementären wissenschaftlichen Revolutionen von Charles Darwin und Karl Marx, die auf den jeweiligen Feldern erstmals eine im emphatischen Sinne geschichtliche Sichtweise etablierten, signifikanter Weise erst nach dem Ende der sogenannten Sattelzeit vollzogen worden sind, was die Erarbeitung zeittheoretischer Binnendifferenzierungen (etwa die Unterscheidung von Verzeitlichung und Vergeschichtlichung) nahelegt. ${ }^{41}$ Probleme und Fragen ergeben sich auch in Bezug auf das Verhältnis der leitenden heuristischen Kategorien der »Geschichtlichen Grundbegriffe« zum 20. Jahrhundert oder in Bezug auf die gleichsam übersprungene oder konzeptuell hinter dem großen Bogen der Modernetheorie zum Verschwinden gebrachte Epoche des Nationalsozialismus. Eine Begriffsgeschichte des 20. Jahrhunderts wird im Gegensatz zu den »Geschichtlichen Grundbegriffen « mit vielfachen Erfahrungsumbrüchen, Schwellen und ungleichzeitigen Entwicklungen rechnen müssen (was Tendenzen der Entgeschichtlichung oder Entpolitisierung einschließt).

III

Zum Abschluss soll nun auf der Basis des Entwickelten die eingangs gestellte Frage nach der Aktualität und den Grenzen von Kosellecks Begriffsgeschichte diskutiert werden. Es kann kaum ein Zweifel daran bestehen, dass die von Koselleck als für die Semantik der Moderne zentral angesehenen Kategorien der Verzeitlichung und Beschleunigung ihre Relevanz behauptet haben, wie vor allem die neuen Prozesskategorien mit der Endung -isierung (engl.: -ication) sowie auch andere neue Kollektivsingulare wie Innovation ver-

40. Geulen, »Plädoyer für eine Geschichte der Grundbegriffe des 20. Jahrhunderts«, S. 91.

41. Vgl. dazu F. Schmieder, »Die wissenschaftlichen Revolutionen von Charles Darwin und Karl Marx und ihre Rezeption in der Arbeiterbewegung «, in Der sich selbst entfremdete und wiedergefundene Marx, hg. v. H. Lethen, B. Löschenkohl und F. Schmieder, Fink, München 2010, S. 39-56. 
deutlichen, die an traditionelle Kollektivsingulare wie Fortschritt oder Revolution anschließen und einige ihrer Funktionen übernehmen. ${ }^{42}$ Die Beschleunigung der Geschichte führt jedoch auch zu einer Verkürzung der historischen Tiefendimensionen der Begriffe sowie zu einer beschleunigten Prägung neuer Begriffe, was zur Tendenz einer Enthistorisierung bzw. zu einer Entgeschichtlichung von Geschichte und einer Annäherung von Begriffsgeschichte und Zeitgeschichte führt. Diese vielfältigen, keineswegs abgeschlossenen Verschiebungen bedeuten aber keineswegs, dass, wie etwa Christian Geulen oder Hans Ulrich Gumbrecht in ihren Thesen zur Verräumlichung oder zur Totalisierung der Gegenwart behaupten ${ }^{43}$, die für die klassische Moderne charakteristische Spannung zwischen Erfahrungsraum und Erwartungshorizont und mit ihr die Bedeutung der Kategorie der Zukunft verschwunden wäre. ${ }^{44}$ Viel eher dürfte es um einen Formwandel der Bearbeitung von Zukunft in der Gegenwart gehen. ${ }^{45}$ Für Koselleck war es der Utopiebegriff, der die Differenz zwischen Erfahrungsraum und Erwartungshorizont ausgemessen hat; heute haben wir es eher mit verwissenschaftlichten Formen wie Prognosen, Szenarien und Risikokalkulationen zu tun, mit denen die Zukunft bearbeitet wird. Eine zweite Veränderung besteht darin, dass an die Stelle der großen teleologischen Zukunftsentwürfe die Darstellung multipler Zukunftsmöglichkeiten, an die Stelle der einen Zukunft also eine Vielfalt möglicher Zukünfte (im Plural) getreten ist. ${ }^{46}$ Die grundsätzliche Problemkontinuität zeigt sich auch in sozialgeschichtlicher Perspektive, denn die Grundstruktur und das treibende Motiv der kapitalistischen Produktionsweise haben sich nicht verändert. Die von Koselleck für die Zukunft vorausgesagte und mittlerweile eingetretene ökologische Wende des Bewusstseins zeigt vielmehr an, dass die politisch-sozialen Konflikte und die Probleme der gesellschaftlichen Naturbeziehungen fortexistieren und sich vielfach erheblich verschärft haben. Wir teilen also immer noch das Bewusstsein Kosellecks, dass die Begriffsgeschichte der Moderne das sprachliche Material einer Epoche untersucht, deren Zukunftsfähigkeit in Zweifel steht und deren entfesselte Dynamik die Lebensbedingungen gefährdet. Hart-

42. Vgl. B. Godin, Innovation Contested. The Idea of Innovation over the Centuries, Berghahn, New York/ London 2015; Susanna Weber, Innovation. Zur Begriffsgeschichte eines modernen Fahnenworts, Tectum, Baden-Baden 2018.

43. Vgl. H. U. Gumbrecht: »Die Gegenwart wird (immer) breiter«, in Merkur. Zeitschrift für europäisches Denken, H. 629/630 (2001), S. 769-784.

44. An anderer Stelle habe ich gezeigt, dass dies auch für das geschichts- und sozialtheoretische Interpretament der Ungleichzeitigkeit des Gleichzeitigen gilt, das für Koselleck von zentraler Bedeutung war; vgl. dazu F. Schmieder: »Gleichzeitigkeit des Ungleichzeitigen. Zur Kritik und Aktualität einer Denkfigur«, in Zeitschrift für kritische Sozialtheorie und Philosophie, Bd. 4, H. 1-2 (2017), S. 325-363.

45. Dies legt auch Lucian Hölscher nahe. Während er in der 1999 erschienenen ersten Auflage seiner sprachgeschichtlich orientierten Untersuchung des Zukunftsbegriffs im Anschluss an zeitgenössische Thesen noch die Auffassung eines Niedergangs der Zukunftserwartungen vertreten hatte, wird diese Auffassung in der 17 Jahre später veröffentlichten zweiten Auflage zurückgenommen; vgl. L. Hölscher, Die Entdeckung der Zukunft, Wallstein, Göttingen 22016, bes. S. 324-326.

46. Vgl. Hölscher, Die Entdeckung der Zukunft, S. 325. 
mut Rosa, der vor einigen Jahren im Rahmen einer umfassenden Studie den Begriff der Beschleunigung sozialtheoretisch und historisch untersucht hat, kommt dann auch zu einer These, die sich in ähnlicher Form bereits bei Koselleck findet. Heißt es bei Koselleck, »dass bestimmte Beschleunigungsvorgänge in unserer ausdifferenzierten Gesellschaft ihren Sättigungsgrad erreicht haben« und »an eine absolute Grenze stoßen müssen« bzw. "sich zunehmend selbst blockieren « ${ }^{47}$, so formuliert Rosa die Vermutung, "dass die in der Moderne konstitutiv angelegte soziale Beschleunigung in der ,Spätmoderne' einen kritischen Punkt übersteigt, jenseits dessen sich der Anspruch auf gesellschaftliche Synchronisation und soziale Integration nicht mehr aufrechterhalten lässt. « ${ }^{48}$ Die Artikulation der Erfahrung, an einer Schwelle der Zeiten zu stehen, dokumentiert gerade durch ihr temporales Zäsurbewusstsein die Abhängigkeit vom Moderneparadigma, das viele neuere Interpreten grundsätzlich verabschiedet sehen. Der zeitliche Abstand, der uns heute von Koselleck trennt, ist in semantischen Studien auszumessen - die Differenzen erscheinen aber keineswegs so tiefgreifend, dass von einer zweiten Sattelzeit zu sprechen ist ${ }^{49}$, was implizieren würde, dass uns die überkommenen Zeitmuster und Semantiken der Moderne so fremd geworden sind, dass sie gleichsam wie eine fremde Sprache übersetzt werden müssen. Bei aller zeitlichen Distanz bleibt Koselleck unser Zeitgenosse, der sich an Problemen abgearbeitet hat, die immer noch ungelöst sind, und dessen theoretische Arbeiten zur modernen Zeitlichkeit und methodische Überlegungen zur Begriffsgeschichte auch weiterhin orientierend und produktiv sind, auch wenn zugleich neue Fragestellungen hinzugekommen sind, mit denen wir allein fertig werden müssen.

47. Koselleck, »Zeitverkürzung und Beschleunigung«, S. 199.

48. Rosa, Beschleunigung. Die Veränderung der Zeitstrukturen in der Moderne, S. 49-50.

49. Vgl. zur Diskussion P. Nolte, »Vom Fortschreiben und Umschreiben der Begriffe«. 